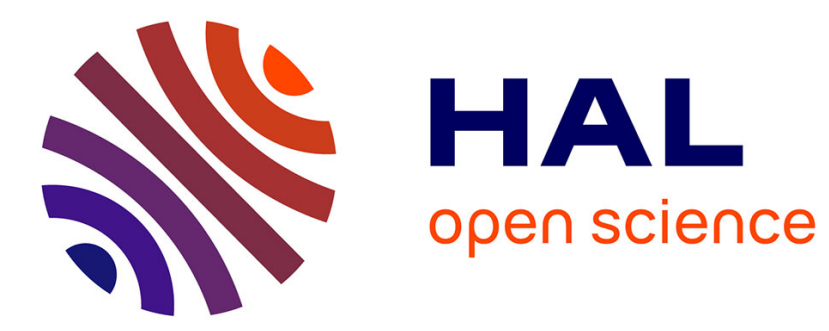

\title{
Pyramide des âges et gestion des ressources humaines
} Eric Godelier

\section{To cite this version:}

Eric Godelier. Pyramide des âges et gestion des ressources humaines. Vingtième siècle. Revue d'histoire, 2007, 3 (95), pp.127-142. hal-00263307

\section{HAL Id: hal-00263307 https://hal.science/hal-00263307}

Submitted on 22 Apr 2008

HAL is a multi-disciplinary open access archive for the deposit and dissemination of scientific research documents, whether they are published or not. The documents may come from teaching and research institutions in France or abroad, or from public or private research centers.
L'archive ouverte pluridisciplinaire HAL, est destinée au dépôt et à la diffusion de documents scientifiques de niveau recherche, publiés ou non, émanant des établissements d'enseignement et de recherche français ou étrangers, des laboratoires publics ou privés. 
chercher : repérer : avancer

Cet article est disponible en ligne à l'adresse :

http://www.cairn.info/article.php?ID REVUE=VING\&ID_NUMPUBLIE=VIN 095\&ID_ARTICLE=VING 0950127

\title{
Pyramide des âges et gestion des ressources humaines
}

\author{
par Éric GODELIER
}

\section{| Presses de Sciences Po | Vingtième siècle}

\section{$2007 / 3-N^{\circ} 95$}

ISSN 0294-1759 | pages 127 à 142 conditions générales d'utilisation du site ou, le cas échéant, des conditions générales de la licence souscrite par votre établissement. Toute autre reproduction ou représentation, en tout ou partie, sous quelque forme et de quelque manière que ce soit, est interdite sauf accord préalable et écrit de l'éditeur, en dehors des cas prévus par la législation en vigueur en France. Il est précisé que son stockage dans une base de données est également interdit. 


\title{
Pyramide des âges et gestion des ressources humaines
}

\author{
Éric Godelier
}

L'utilisation de la pyramide des âges dans les discours des directeurs de ressources humaines s'est développée au cours des années 1980. Elle renvoie au choix d'un critère de sélection de salariés jugés en sureffectif. Derrière l'âge et ses représentations, c'est bien la question de la capacité des salariés à s'adapter au changement de l'entreprise qui est posée. En définitive, il s'agit de savoir s'il existe des conditions démographiques au changement organisationnel.

Au début des années 1990, les groupes Renault et PSA ont sollicité les pouvoirs publics afin qu'ils les aident à rééquilibrer leur pyramide des âges. Déjà en 1988, c'est pour les mêmes raisons qu'Usinor-Sacilor, devenu Arcelor en 2001, mettait fin à la convention générale de protection sociale (CGPS) organisant les départs en préretraite. Cette décision lui permettait de reprendre une politique d'embauches ${ }^{1}$. La pyramide des âges apparaît régulièrement dans le discours des dirigeants comme un outil central de la stratégie de restructuration des ressources humaines que mettent en œuvre de plus en plus d'entreprises. Elle est devenue un des instruments de référence dans la construction d'une gestion prévisionnelle des emplois et des compétences $(\mathrm{GPEC})^{2}$. Pourquoi cet intérêt crois-

(I) Francis Mer, « Il va falloir débaucher pour embaucher », Liaisons sociales, 42, octobre 1989, p. 34-36. Cf. Éric Godelier, Du capitalisme familial à la stratégie de groupe : le cas de l'usine sidérurgique de Montataire, Paris, Éd. de l'EHESS, 1990.

(2) Loïc Cadin, Francis Guérin et Frédéric Pigeyre, Gestion des ressources humaines, pratiques et éléments de théorie, Paris, Dunod, 1999. sant pour la pyramide des âges dans les entreprises ? Comment l'âge intervient-il dans la stratégie de gestion des ressources humaines ? Y a-t-il des conditions démographiques au changement organisationnel ? Cet article se propose de faire le point sur cet outil et sur les conditions de son utilisation.

Il s'agit premièrement d'étudier la façon dont les responsables d'entreprises - dirigeants, direction des ressources humaines, collaborateurs de la fonction personnel ou membres de la ligne hiérarchique de tous niveaux - ont mobilisé l'âge comme élément de construction d'un processus de décision et de pilotage des ressources humaines ${ }^{3}$. La mobilisation de l'âge par les décideurs cache souvent une interrogation sur les possibilités d'un changement organisationnel. Elle se traduit encore très fréquemment par une stratégie d'élimination des salariés les plus âgés ${ }^{4}$. Les dirigeants construisent donc un modèle des éventuelles sources de blocages ou d'inertie, qui pourraient empêcher leur entreprise de s'adapter aux nouvelles conditions de l'environnement. Plus généralement, ces abrégés de gestion qui définissent ce qui est bon et

(3) Jean Fombonne, Personnel et DRH. L'affirmation de la fonction personnel dans les entreprises (France, 1830-1990), Paris, Vuibert, 2001. Il va sans dire que ce regroupement hétérogène d'acteurs ne vise pas à nos yeux à occulter que chacun d'entre eux est soumis à des contraintes et des enjeux qui lui sont spécifiques. En particulier, la contribution à la construction de l'outil et l'utilisation de la pyramide des âges sont bien évidemment variables selon leur spécialité et leur position dans la hiérarchie de l'entreprise.

(4) Gilles Guérin, « La gestion du vieillissement : un bilan », Revue de gestion des ressources humaines, 2, décembre 1991-janvier 1992, p. 3-27. 
bien pour conduire l'entreprise ${ }^{1}$ sont largement influencés par les représentations de l'âge que notre culture ou notre société construisent et valident ${ }^{2}$. Celles-ci sont souvent cristallisées par et dans la construction d'un outil statistique utilisé dans les discours sur l'âge : la pyramide des âges. Or ces représentations, bien qu'approximatives, sont rarement critiquées par les dirigeants et continuent de fonder de nombreuses décisions en matière de gestion des ressources humaines : embauches, promotions, mobilités, départs, etc. Elles reposent pourtant sur des a priori qui amalgament l'âge et la diminution des capacités d'un individu. Faut-il y voir un autre de ces mythes managériaux ${ }^{3}$ ? Alors que se développent depuis deux décennies des stratégies de flexibilisation organisationnelles et commerciales dans la plupart des grandes entreprises, il s'agit bien de comprendre ce qui, éventuellement, entraîne une « rigidification » des salariés à mesure que le temps passe.

Un deuxième aspect de la question renvoie à la façon dont la pyramide des âges, à l'origine instrument de la démographie, est construite dans certaines entreprises puis transformée en outil de pilotage de la gestion des ressources humaines. Ce transfert entre deux domaines distincts contribue lui-même à valider certaines représentations de l'âge évoquées précédemment. En particulier, il faut comprendre comment les décideurs passent, souvent implicitement, de la constatation d'un simple déséquilibre statistique entre les tranches d'âges des

(I) Claude Riveline, « Un point de vue d'ingénieur sur la gestion des organisations », Annales des Mines. Gérer et Comprendre, décembre 1991, p. 50-62.

(2) Marie-Annick Déjean de la Batie, « La transmission des connaissances en formation gérontologique », Gérontologie et Société, 46, 1988 (l'auteur remercie Mme Catherine Dumoutier de la Fondation nationale de gérontologie pour son aide) ; Sylvie Le Minez, «Les entreprises et le vieillissement de leur personnel : faits et opinions », Travail et Emploi, 63, février 1995, p. $23-40$.

(3) James March, «Les mythes du management », Annales de l'École de Paris, 5, 1998, p. 387-394. salariés, à l'élaboration d'un discours sur le changement et sur la relation entre âge et vieillissement et capacités de travail, alors même que de nombreux travaux prouvent que cette dernière est beaucoup plus complexe qu'il n'y paraît. Ce glissement entraine des erreurs de diagnostic et aboutit parfois à la mise en place de solutions inadaptées. Dans le même temps, un autre outil d'analyse statistique issu de la démographie, la cohorte de population, semble proposer aux gestionnaires une approche plus fine des phénomènes de vieillissement.

Pourquoi les discours et certaines pratiques en matière de rééquilibrage des pyramides d'âges semblent converger, alors même que de nombreuses études montrent que la gravité des déséquilibres est extrêmement variable ${ }^{4}$ ? Le recours à la pyramide paraît bien être une métaphore qui masque certains des enjeux profonds liés au changement : modification des fondements de l'autorité et de la légitimité des différents niveaux hiérarchiques, éclatement des systèmes de promotion à l'ancienneté, développement de la polyvalence, accroissement de la compétence, réduction des sureffectifs, etc.

\section{Émergence d'un discours}

Dans quel contexte et de quelle manière la question du déséquilibre entre les tranches d'âge des salariés est-elle progressivement devenue un point de référence de la gestion des ressources humaines ? Le recours à la pyramide des âges s'inscrit historiquement dans le débat sur la détermination de l'âge de la retraite. Il correspond ensuite à une prise de conscience de l'inadéquation de la stratégie de gestion des ressources humaines aux transformations de l'environnement. Ici, deux thèmes reviennent de façon récurrente : la nécessaire résorption

(4) Olivier Galland, Jocelyne Gaudin et Philippe Vrain, « Contrats de solidarité de préretraite et stratégie d'entreprises », Travail et Emploi, 22, décembre 1984, p. 7-20. 
d'un sureffectif et la mise en place d'un changement dans l'organisation du travail ${ }^{1}$.

\section{Évolutions}

L'évolution est d'abord politique et conventionnelle. Les premières interrogations relatives à l'influence de l'âge sur la performance des entreprises sont apparues durant la décennie 1980. Elles prolongent à l'intérieur de l'entreprise des débats politiques et sociaux qui, depuis le début des années 1970, agitent les partenaires sociaux et les pouvoirs publics à propos de l'âge de la retraite et des modes de vie de la vieillesse. L'année 1972, avec la préparation de l'accord interprofessionnel sur la garantie de ressources aux chômeurs âgés ${ }^{2}$, marque un tournant dans cette évolution ${ }^{3}$. Plusieurs problèmes sont débattus : l'abaissement de l'âge de la retraite, l'amélioration des conditions de travail pour éviter l'usure prématurée des salariés ou l'amélioration des conditions de vie des retraités. Cet accord prévoit la possibilité de départs avant l'âge légal de la retraite. En contrepartie, il organise une garantie de ressources aux chômeurs âgés. Durant la période qui suit, la crise et les restructurations font progressivement émerger un accord tacite sur l'idée que les départs en retraite permettent de créer des emplois. Alors qu'il s'agit de deux phénomènes différents ${ }^{4}$, le chômage (c'est-à-dire l'envers du travail) et la retraite (le non-travail) se trouvent rapprochés au sein d'accords paritaires et de politiques de

(I) Parmi les thèmes les plus souvent évoqués, on retrouve le changement de technologie, le passage à une politique de qualité totale, la mise en place d'une organisation du travail plus qualifiante ou l'abandon d'une gestion taylorienne du personnel reposant sur des postes prescrits.

(2) Accord du 27 mars confirmé par la loi du 5 juillet 1972.

(3) Anne-Marie Guillemard, « La dynamique sociale des cessations anticipées d'activité », Travail et Emploi, 15, janviermars 1983, p. 15-31.

(4) Même si, comme le montre Jacques Freyssinet, les frontières entre l'un et l'autre tendent à devenir poreuses. (Jacques Freyssinet, Le Chômage, Paris, La Découverte, « Repères », 1987, p. 19) lutte pour l'emploi. De la fin des années 1960 à 1982, se multiplient les accords d'entreprises ou de branches qui organisent les possibilités de départs en préretraite. De fait, sinon de droit, l'âge devient une variable de sélection des salariés, d'abord vers le licenciement puis, après $1977^{5}$, vers la préretraite. Deux concepts se construisent alors l'un l'autre : l'« employabilité » et le $\ll$ retraitable ${ }^{6}$.

L'évolution est aussi économique. Les années 1970-1980 marquent pour les entreprises une diminution globale de la demande et la transformation de l'environnement commercial et technique. Les directions générales commencent à s'interroger sur les éléments à modifier : quel nouveau modèle pour l'entreprise efficace ? Que faire pour répondre aux nouvelles contraintes posées par l'environnement : une concurrence par les prix de plus en plus rude et la recherche d'une plus grande flexibilité dans l'organisation et les modes de gestion ${ }^{7}$ ? Il leur faut réaliser des gains de productivité et concevoir des structures plus souples et plus légères. Dans la décennie 1980, les entreprises généralistes adoptent des stratégies de recentrage et de réduction des effectifs. Face à un environnement changeant, c'est le modèle de l'entreprise spécialisée qui paraitt l'avoir emporté. Également mis en cause sont les éventuels facteurs d'inertie structurelle qui freinent le changement ${ }^{8}$. La question se pose alors de savoir si une entreprise peut être efficace avec des salariés âgés ou si elle sera éliminée du marché, ce qui revient à se demander s'il y a des conditions démographi-

(5) Accord du 13 juin donnant une garantie de ressources aux démissionnaires âgés.

(6) Xavier Gaullier et Maryvonne Gognalons-Nicolet, « Crise économique et mutations sociales. Les cessations anticipées d'activité (50-65 ans) », Travail et Emploi, 15, janviermars 1983, p. 33-45.

(7) Robert Boyer, La Flexibilité du travail en Europe, Paris, La Découverte, 1987.

(8) Michael Hannan et John Freeman, « Structural Inertia and Organizational Change », American Sociological Review, 49, avril 1984, p. 149-164. 
ques au changement et à l'efficacité organisationnelle. Dans un tel contexte, les entreprises doivent définir des critères pour sélectionner les personnes en sureffectif.

\section{Définir un critère de tri}

Le choix d'un critère de tri se cristallise sur la variable « âge ». Résorber le sureffectif suppose de définir quantitativement et qualitativement les catégories de salariés qu'on juge inutiles ou inadaptées aux nouvelles conditions de production. Celles qui sont jugées trop « vieilles » paraissent « logiquement » devoir faire partie de ce groupe. La question de l'équilibre de la pyramide des âges contribue alors implicitement à construire la notion de sureffectif ${ }^{1}$. Le diagnostic se diffuse par mimétisme assez rapidement entre entreprises d'un même secteur ou d'une même région, sans d'ailleurs que la réduction d'effectifs soit toujours justifiée du point de vue économique ${ }^{2}$.

L'approche est d'abord quantitative. Comment réduire la masse salariale en supprimant un certain nombre d'unités au sein de l'effectif salarié ? Du point de vue strictement statistique, chaque salarié est supposé interchangeable. Un tel raisonnement montre ses limites car les salariés sont aussi porteurs de compétences et d'expérience. Il apparaît contradictoire avec les discours qui valorisent l'importance des hommes dans l'entreprise. Rapidement, la sélection d'une population nécessite d'introduire d'autres éléments plus qualitatifs, c'est-à-dire de mieux prendre en compte la question du contenu et des nouvelles conditions de la productivité dans l'entreprise. Ce sont les besoins futurs en termes de compétences qu'il faut au préalable évaluer pour pouvoir définir ensuite le sureffectif. Le critère de sélection est alors celui des compétences jugées irrémédiablement inutiles pour

(I) Louis Mallet, « Les sureffectifs dans l'entreprise : analyse et enjeux », Problèmes économiques, 2152, 1989, p. 15-27.

(2) Rachel Beaujolin, Les Vertiges de l'emploi. L'entreprise face aux réductions d'effectifs, Paris, Grasset, 1999. l'avenir de l'entreprise. Ce faisant, les notions de déséquilibre de la pyramide des âges et de sureffectif deviennent plus difficiles à manier, car la question de l'âge s'efface derrière le problème de la gestion des compétences. Or, ce ne sont pas forcément les plus âgés qui sont les moins compétents ! Dans ces conditions, il n'y a donc plus de déterminisme entre déséquilibre des tranches d'âges, déséquilibre dans la répartition des compétences et inertie structurelle. Néanmoins, le raisonnement fondant la détermination du sureffectif sur l'âge suppose implicitement que les salariés les plus âgés ne peuvent s'adapter individuellement aux nouvelles conditions de la production, ce qui empêcherait la modernisation de l'entreprise. D'où provient un tel amalgame? Un rapide détour pour préciser la problématique du changement dans l'entreprise est ici nécessaire.

Le plus souvent, c'est au moment où les dirigeants développent une nouvelle approche de la productivité de l'entreprise que le retour à l'équilibre de la pyramide des âges est sollicité pour légitimer l'éventuel départ des salariés âgés. En simplifiant, il s'agit de passer d'une logique reposant sur une maximisation locale de l'efficacité, qu'on pourrait qualifier de taylorienne, à une logique globale, flexible, où la productivité est obtenue globalement à l'échelle organisationnelle à l'aide d'une gestion plus étroite et plus flexible des flux de produits. Dans la forme «taylorienne », le contenu technique et le rythme de production sont donnés par l'outil. Le poste de travail - en particulier pour les opérationnels - impose des contraintes physiques, psychologiques et intellectuelles strictes à son titulaire. Historiquement, cela a permis l'embauche de personnel rapidement opérationnel dont la formation initiale était faible ${ }^{3}$. Au contraire, la nouvelle logique organisation-

(3) Bernard Lutz, Le Mirage de la croissance marchande, Paris, Éd. de la MSH, 1990. 
nelle valorise beaucoup plus le concept de productivité «collective », c'est-à-dire les relations entre des postes de travail au contenu enrichi et élargi. On comprend dès lors pourquoi, de proche en proche, tous les salariés âgés de l'entreprise sont concernés par le changement. Cette analyse s'appuie sur l'hypothèse de besoins de changement identiques dans l'ensemble des services de l'entreprise. Or, dans de nombreux cas, rien ne permet a priori de l'affirmer ${ }^{1}$. Au contraire, le vieillissement des effectifs reste extrêmement variable selon les entreprises et les catégories de salariés. Si l'âge moyen augmente en France entre 1979 et 1990 pour les ouvriers et les employés techniciens et agents de maitrise (ETAM), il ne varie pas chez les ingénieurs et cadres. En outre, il diminue dans certains secteurs comme le commerce alimentaire ou de détail. Enfin, ce sont les entreprises de trois cents à cinq cents salariés qui sont les plus touchées.

Il y a parfois un trompe-l'œil statistique, car ce vieillissement provient surtout d'un ralentissement de l'embauche des jeunes dû à la crise ${ }^{2}$. La pyramide des âges semble donc ne pas constituer le meilleur outil de diagnostic pour le gestionnaire enclin à se pencher sur les questions de vieillissement de son personnel. Pourtant, son usage se diffuse dans l'entreprise. Pour saisir ce phénomène, il faut s'interroger sur la relation que cherchent à construire certains dirigeants ou directeurs des ressources humaines entre âge, vieillissement et diminution des capacités de travail et d'adaptation : « À quel âge est-on trop vieux ${ }^{3}$ ? »

(I) Géraldine Schmidt, « Les logiques d'action des entreprises à l'égard de l'âge. Analyse monographique de six établissements », Travail et Emploi, 63 (2), février 1995, p. 41-57.

(2) José Allouche, « Lâge et l'ancienneté des salariés de 255 bilans sociaux d'entreprise (1979-1990) », Revue de gestion des ressources bumaines, 7 , mai 1993 , p. 37-48.

(3) Catherine Teiger et Robert Villatte, « Conditions de travail et vieillissement différentiel », Travail et Emploi, 16, avril-juin 1983 , p. $27-36$

\section{Définir le salarié «âgé»}

Le discours et les pratiques en matière de gestion des salariés âgés reposent sur des représentations du vieillissement où les capacités de chacun diminuent de façon homogène et irréversible dans le temps. Comment l'entreprise et la gestion se sont-elles approprié ces représentations ? Qu'entendent-elles par «salarié âgé » ? Deux types d'argumentations sont utilisées plus ou moins explicitement dans la gestion du personnel : l'âge représente et résume le vieillissement; le vieillissement se traduit par une diminution continue des capacités individuelles.

Concernant le premier point, Xavier Gaullier montre clairement que la notion de « salarié âgé » varie historiquement selon les besoins de sélection des entreprises et l'état du marché du travail : «En période de croissance, de pleinemploi et de pénurie de main-d'œuvre, le salarié âgé est un producteur efficace qu'on cherche à retenir ; avec les crises économiques, le chômage et les sureffectifs, le salarié âgé devient un incapable dont il faut se séparer par tous les moyens. » Il ajoute : « L'âge apparaît divers et changeant selon les opinions et les intérêts. Il est ainsi un concept-écran qui masque d'autres réalités : la génération, le sexe, la situation historique. [...] Le "salarié-âgé" est bien une production sociale et cela est lourd de conséquences [...]. Dès lors l'âge devient directement ou indirectement un critère de gestion et de sélection dans l'entreprise. [...] Il amène les individus à intérioriser leur propre dévalorisation ${ }^{4}$. »

Une telle variété dans la signification prêtée à l'âge selon les conjonctures amène à douter du contenu d'un concept aussi peu stabilisé. Elle souligne le risque attaché à la mobilisation de l'âge comme variable objective de pilotage de la gestion des ressources humaines. Cette démarche minimise d'autres éléments de l'histoire

(4) Xavier Gaullier, «Qu'est-ce qu'un salarié âgé ? », Gérontologie et Société, 45, 1988, p. 115-127. 
et de l'évolution des salariés : l'accumulation de compétences, des savoir-faire et de l'expérience, l'influence des règles conventionnelles dans la construction des carrières individuelles, le rôle de la technologie ou des mécanismes de régulation concurrentielles dans la constitution d'une compétence globale de l'organisation. En réduisant l'évolution professionnelle à l'âge, l'entreprise détache les critères de sélection et d'évaluation des compétences individuelles mises en œuvre dans le travail pour les faire peser sur la personne du salarié, non seulement résumée par son seul âge, mais aussi parfois par des événements qui lui sont associés (possibilité de naissance des enfants, situation matrimoniale ou patrimoniale, etc.). L'âge devient, pour certains responsables de ressources humaines, un critère, conscient ou non, d'intégration ou d'exclusion dans les processus de gestion : le recrutement dépend de l'âge d'une éventuelle future « mère »; l'envoi en formation se fait de plus en plus rare pour les salariés âgés; des retards ou des blocages apparaissent dans la promotion, ou encore des affectations à des postes déclassés ${ }^{1}$. En réponse, certains salariés - en particulier les moins qualifiés - développent des stratégies de repli sur eux-mêmes qui anticipent leur exclusion à terme de l'entreprise. Il leur semble impossible de lutter contre l'avancée inexorable de l'âge, ce qui les amène à s'autostigmatiser $^{2}$. Ils refusent ce que peut leur proposer l'entreprise en matière de formation ou de promotion et accélèrent un peu plus leur dévalorisation individuelle et collective ${ }^{3}$. Chez d'autres, on voit apparaître une stratégie de dis-

(I) Dominique Dessors, Jean Schram et Serge Volkoff, « Du "handicap de situation" à la sélection-exclusion », Travail et Emploi, 48, février 1991, p. 31-47.

(2) Erwing Goffman, Stigmate, les usages sociaux des bandicaps, Paris, Éd. de Minuit, 1989.

(3) Éric Godelier, Usinor-Arcelor : du local au global, Paris, Hermes, 2006 ; Gwénaèlle Poilpot-Rocaboy, «Performance de la gestion des emplois : éviction ou rétention des salariés vieillissants ? », in Actes du 7e Congrès annuel de l'AGRH, Paris, Association de gestion des ressources humaines, 1996, p. 423-436. simulation de certaines difficultés physiques ou mentales qui les amène à « tenir coûte que coûte » pour satisfaire aux contraintes imposées par le poste, et accélère encore la diminution de leurs capacités ${ }^{4}$.

Le terme de « salarié âgé » dans l'entreprise ne paraît pas nécessairement le meilleur pour traiter du problème de gestion des sureffectifs et du changement. En évoquant le problème du déséquilibre de la pyramide des âges, certaines entreprises ne seraient-elles pas plutôt à la recherche d'un nouveau type de salarié, plus à même de s'adapter à de nouvelles conditions de travail ? Si les représentations des relations entre l'âge, le vieillissement et les capacités sont pour le moins extrapolées, comment expliquer les relations que tissent depuis les années 1980 les dirigeants de certaines entreprises entre l'âge, le vieillissement et les possibilités de changements individuels ou organisationnels ? Autrement dit, comment expliquer que les salariés âgés deviennent « vieux »?

La relation entre l'âge, le travail et le vieillissement ne peut être appréhendée sans l'introduction de l'organisation du travail et de son rôle de médiateur entre l'âge du salarié et la mise en œuvre de ses capacités. De ce point de vue, c'est bien l'entreprise qui dispose des moyens pour organiser le milieu et le contenu du travail. Elle contribue donc plus ou moins directement à définir les conditions du vieillissement des salariés. À l'inverse, elle peut soulager les contraintes qui pèsent sur lui. Il convient alors de préciser dans cette nouvelle approche ce à quoi correspond exactement un salarié âgé.

Le vieillissement est « l'évolution défavorable à l'organisme qui altère les équilibres biologiques, psychologiques et sociaux et qui apparaît constante et irréversible ${ }^{5} \gg$. Ainsi un

(4) Dominique Dessors, Jean Chram et Serge Volkoff, op. cit.

(5) Henri Péquinot, Vieillir et être vieux, préf. de Georges Canguilhem, Paris, Vrin, 1981. 
« vieux »-terme souvent péjoratif - ou un salarié âgé serait un individu qui ne pourrait ou ne voudrait plus s'adapter aux conditions de vie que lui impose son environnement, à savoir ici l'organisation du travail conçue et mise en œuvre par l'entreprise. Dans ce cadre, il y a deux types d'approches des relations entre l'âge et le vieillissement des salariés ${ }^{1}$. La première est l'usure par le travail. Comme toute activité, le travail fatigue, entame les capacités, amoindrit la résistance ${ }^{2}$. C'est sur ce point que se cristallisent les déterminismes évoqués plus haut entre âge et diminution des capacités. Dans ce cas, en particulier pour les tâches pénibles, c'est la longueur d'exposition, et non l'âge, qui accélère ou au contraire ralentit le vieillissement.

La seconde est le vieillissement par rapport au travail, c'est-à-dire l'adaptation du salarié dans sa relation au travail. Ici, l'évolution de ses capacités individuelles dépend non seulement de l'effet normal du temps, mais aussi des contraintes posées par l'organisation du travail (postures physiques, attention mentale, cadences de production, rythmes et durée de présence). Dans la première approche, c'est le salarié qui est seul « responsable » de son inadaptation à un travail, l'organisation étant neutre ; dans la seconde, l'inadaptation a des raisons physiologiques et organisationnelles. Plus que l'âge, c'est ici l'organisation qui fait donc vieillir les salariés!

Jusqu'au début des années 1990, l'essentiel des entreprises ont utilisé des principes tayloriens d'organisation du travail reposant sur une parcellisation et spécialisation étroite des tâches; de nombreux postes où le travail se déroule suivant des procédures strictes ou sous

(I) Serge Volkoff, Antoine Laville et Marie-Christine Maillard, « Âges et travail : contraintes, sélection et difficultés chez les 40-50 ans », Travail et Emploi, 54, avril 1992, p. 21-33.

(2) Maurice de Montmorillon, L'Ergonomie, Paris, La Découverte, « Repères », 1986. contrainte de temps ; un recrutement de personnel à faible qualification pour les tâches d'exécution ; une politique de formation essentiellement destinée aux salariés les plus qualifiés ; une politique de promotion reposant en général sur l'ancienneté ${ }^{3}$. En les cantonnant dans des situations de travail répétitives, parcellisées et très rigides, ce type d'organisation a bloqué les possibilités d'apprentissage en réduisant la variété et la complexité des situations rencontrées. Or c'est précisément la variété et le renouvellement des situations lors de la vie professionnelle qui préparent l'individu à son adaptation ultérieure. En outre, le peu de marges laissé aux salariés s'est révélé handicapant lorsque ceux-ci ont été confrontés aux problèmes « normaux » de l'âge ${ }^{4}$. Il en a découlé un « vieillissement » pour de nombreuses catégories de salariés : ouvriers, employés administratifs, techniciens et agents de maîtrise. De ce point de vue, l'organisation taylorienne a ellemême créé les conditions de son inadaptation future, en rigidifiant les méthodes de production et les salariés qui y étaient exposés, c'està-dire pour l'essentiel les opérationnels les moins qualifiés. Progressivement adaptés à l'organisation taylorienne, ils paraissent peu flexibles pour les nouvelles règles de l'organisation, en particulier dans leurs capacités d'adaptation à de nouveaux emplois et à de nouvelles compétences.

Ainsi, si Usinor n'a pas mis en place une organisation aussi taylorisée que celle de l'industrie automobile par exemple, la logique d'optimisation économique locale, centrée selon les cas sur chaque poste de travail ou sur une unité (service de production, usine), a fortement marqué le modèle économique jusqu'aux années

(3) Armelle Gorgeu et René Mathieu, « Nouvelles usines : nouvelle gestion des emplois ? », La Lettre du Centre d'études de l'emploi, 36, février 1995, p. 1-10.

(4) Dominique Dessors, Jean Schram et Serge Volkoff, op. cit. 
1980 ․ Longtemps, la progression des salariés a été organisée non seulement en les fixant dans une seule usine, mais aussi à l'intérieur d'un même atelier. Après la crise de 1974, les restructurations et la quasi-nationalisation de 1978 aboutissent à un éclatement de ces anciennes logiques. L'effondrement des prix et de la demande conduit rapidement l'entreprise au bord de la faillite. À partir de cette période, de nombreuses installations sont fermées : Valenciennes, Thionville et bientôt Denain. Ces mesures entraînent de très fortes réactions sociales et politiques dans les régions du Nord et de l'Est de la France. Dès 1977, un plan de préretraite, la convention générale de protection sociale (CGPS) ${ }^{2}$, organise les départs des salariés dans les sites ou les ateliers les plus touchés. Ceux qui sont trop jeunes pour bénéficier de cette disposition se voient proposer une mutation vers d'autres usines maintenues en activité. Ils remplacent les plus âgés qui ont bénéficié de la CGPS. L'État prend l'essentiel des coûts financiers de l'opération à sa charge. En contrepartie, il impose un blocage des embauches qui perdure jusqu'en 199133. En près de quatorze ans, ce sont quatre-vingt-dix-huit mille personnes qui quittent Usinor-Sacilor grâce à ce plan de préretraite. Ce dispositif entraîne un vieillissement progressif du personnel.

Mais le processus est remis en cause par l'État et la commission de Bruxelles à partir de 1988, car l'entreprise redevient bénéficiaire.

(I) De façon rapide, il est possible ici de définir le taylorisme autour de deux axes : une logique d'organisation axée sur la productivité du couple salarié-poste de travail et une spécialisation poussée des tâches et des employés.

(2) Une autre convention, la Convention de protection sociale de la sidérurgie (CPS) est réservée aux ingénieurs et cadres. Dans sa première version les salariés touchaient environ $98 \%$ de leur dernier salaire. On estime que les entreprises sidérurgiques n'ont effectivement supporté que $10 \%$ du coût d'un préretraité, soit $120000 \mathrm{~F}$ (environ $18400 €$ ).

(3) En fait, seules sont autorisées des embauches dites « techniques ». Il s'agit de 500 personnes essentiellement des jeunes diplômés (grandes écoles, BTS/DUT).
L'arrêt de la CGPS entraîne des conséquences paradoxales pour l'entreprise. D'un côté, il permet de lever les contraintes qui pesaient sur l'embauche et de la relancer. De l'autre, il fait surgir des problèmes de gestion du personnel jusque-là résolus par la convention. Le départ des salariés les plus âgés, souvent situés en haut de la hiérarchie, autorisait une progression de carrière rapide et régulière ainsi qu'une augmentation des revenus. La motivation était aussi obtenue par la perspective d'un départ rapide ${ }^{4}$. Or désormais, il faut trouver de nouvelles perspectives de carrières aux salariés de 45 ans qui, du jour au lendemain, voient l'échéance de leur départ passer de cinq à quinze ans ${ }^{5}$. L'innovation technologique amène naturellement la direction générale à s'interroger sur les besoins de formation et le rythme des investissements. Une nouvelle stratégie est donc à mettre en place. L'hypothèse initiale est que la sidérurgie reste encore en sureffectif à la fin des années 1980. En 1989, le président-directeur général Francis Mer suscita de vives réaction dans l'entreprise, en déclarant dans un entretien que pour rééquilibrer la pyramide des âges, «il va falloir débaucher pour embaucher $\gg$. Sa constatation est qu'à l'échéance de l'an 2000, si aucune mesure n'est prise, $50 \%$ de l'effectif auront plus de 50 ans. Il faut donc définir un critère de sélection des salariés en sureffectif. L'effort est demandé aux salariés de la tranche 30-40 ans, ceux qui avaient déjà été confrontés à la crise et à qui promesse avait été faite d'un maintien durable dans l'emploi en contrepartie d'efforts de formation, de mobilité et de mutation. La crispation sociale se réinstalle dans l'entreprise.

(4) 60 ans pour les ouvriers, employés et agents de maîtrise, puis 55 et 50 ans dans la dernière version de la convention.

(5) De nombreux salariés de cet âge avaient tendance à adopter des stratégies d'activité ralentie, parfois combinées à des mises à l'écart organisées de façon plus ou moins consciente par l'entreprise (retrait des propositions de formation, plan de mobilité, etc.) 
Rapidement pourtant, la direction du groupe prend conscience de l'impact négatif de ces déclarations et des limites d'un diagnostic fondé de façon exclusive sur les catégories et représentations trop approximatives issues des critères démographiques. Le constat théorique précédent et l'exemple d'Usinor doivent-ils amener à remiser définitivement l'outil d'analyse et de gestion du personnel que constitue la pyramide des âges?

\section{Usages de la pyramide des âges}

Comment l'entreprise utilise-t-elle un outil démographique pour en déduire des prévisions de nature économique ou organisationnelle ? Ce débat sur l'importation de la démographie dans un autre contexte n'est pas nouveau et soulève de nombreuses difficultés ${ }^{1}$. Deux points méritent ici une attention particulière. D'une part, il convient d'examiner la façon dont s'effectue le transfert d'une constatation de nature démographique à un raisonnement de nature gestionnaire. D'autre part, en tant que construction statistique, la pyramide des âges présente des limites dont l'analyse doit tenir compte, car elles peuvent fausser les conclusions et donc les décisions qui en découlent. En particulier, le vieillissement de l'âge moyen constaté par la pyramide peut avoir plusieurs origines : l'arrêt du rajeunissement du personnel ou son vieillissement réel. Selon le cas, les conséquences sont très différentes pour la gestion de l'entreprise. Sans conscience des difficultés de passage, les solutions élaborées peuvent se révéler coûteuses à long terme, car elles minimisent le rôle d'autres variables du changement comme l'évolution de la technologie ou la formation des différentes catégories de salariés. Une grande part de ces difficultés tient aussi au fait que, dans certaines entreprises, la pyramide des âges fait office d'outil d'analyse du vieillissement et de prospective sur les capacités de changement du personnel : on a évoqué la complexité et les limites de cette relation. D'autres outils semblent se mettre en place comme la cohorte, qui semblent pouvoir pallier certaines insuffisances d'un usage trop approximatif de la démographie.

\section{De la démographie à la gestion}

Deux types d'usages peuvent être faits de la pyramide des âges par les gestionnaires de la direction générale ou des ressources humaines. D’un côté, elle constitue un outil de prévision des départs à la retraite, mais aussi d'anticipation des évolutions en termes d'emplois et de compétences, s'inscrivant ainsi dans la réflexion stratégique des dirigeants de l'entreprise. De l'autre, elle est un outil de politique des rémunérations.

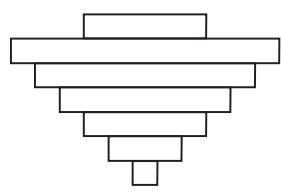

« Champignon »

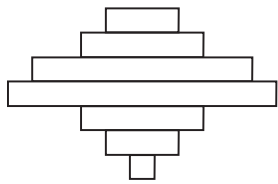

«Toupie»

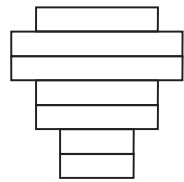

«Cylindre »

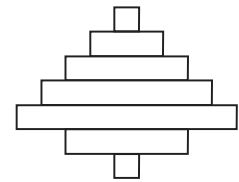

« Poire écrasée »

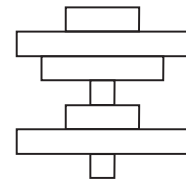

« Pelote de laine »

1. Types de pyramides des âges schématisées.

(I) Patrice Bourdelais, «Vieillissement de la population ou artefact statistique ? », Gérontologie et Société, 49, juillet 1989, p. 23-32. 
Selon la situation démographique de l'entreprise, les possibilités ou les risques stratégiques sont différentes. Dans la pyramide de type « champignon », l'entreprise peut proposer aux salariés des perspectives d'évolution rapide dans des cas de restructurations ou de changements technologiques importants. Elle aura plus de difficultés à motiver les salariés les plus jeunes ou situés en bas de la hiérarchie si l'évolution de la population se fait de façon régulière ${ }^{1}$.

Dans le second cas, « toupie », l'entreprise a de toute évidence massivement embauché pour des raisons conjoncturelles (croissance du marché, développement rapide d'une activité ou d'une technologie, effets d'aubaine de mesures fiscales des pouvoirs publics sur certaines populations). Si l'effectif visé doit rester constant, la situation des catégories de salariés des tranches d'âges les plus nombreuses peut se révéler délicate. Celle des plus jeunes peut aussi poser problème à court terme, surtout si l'entreprise souhaite maintenir un effectif constant. À plus long terme, le départ très rapide des tranches les plus nombreuses ouvre des possibilités d'embauches de jeunes salariés, mais peut aussi entraîner une perte massive de savoir-faire et de compétences. Cette forme permet toutefois de disposer de salariés en surnombre, pour pouvoir construire une politique de carrière.

La forme en «poire écrasée » correspond classiquement à une entreprise ayant cessé de recruter - volontairement ou non - durant de longues années. Le risque de pertes de savoirs et d'expériences est très important. Dans des

(I) Ce raisonnement repose sur l'hypothèse que les salariés entrent dans l'entreprise en bas de la hiérarchie. D'autres politiques de gestion des ressources humaines avec la constitution de marchés internes séparés et cloisonnés supposeraient de construire une pyramide des âges par marché interne ou par catégories de salariés (jeunes, femmes, cadres, ingénieurs...). Voir, par exemple, Bernard Gazier, Les Stratégies de ressources humaines, Paris, La Découverte, « Repères », 1993 ; Dominique Gambier et Michel Vernières, Le Marché du travail, Paris, Economica, 1991. industries de main-d'œuvre ou de hautes technologies, peuvent en résulter des difficultés de production ou de renouvellement des connaissances en matière technologique.

La forme en « pelote de laine » correspond à une des évolutions possibles du type précédent. Elle traduit une stratégie de lutte contre le déséquilibre démographique à l'aide d'embauches massives. Si elle ouvre effectivement des perspectives de promotion rapide pour les nouveaux arrivants, l'entreprise risque néanmoins d'être confrontée à des pénuries dans l'encadrement intermédiaire ou aux premiers échelons des catégories « ingénieurs et cadres ». Cette situation n'est pas automatiquement préjudiciable si elle touche des secteurs où la main-d'œuvre est autonome ou très qualifiée (luxe, ingénierie, artisanat).

Reste la forme en « cylindre », qui marque la plupart du temps une stratégie d'embauche régulière et permet de définir une progression dans la carrière de chacun. Dans tous les cas, cet outil de management stratégique doit évidemment s'accompagner d'une étude de la pyramide des anciennetés du personnel ou des dirigeants. Outre les perspectives en termes de départs à la retraite, d'emplois ou de compétences, les questions démographiques résumées dans la pyramide des âges ont clairement des impacts financiers, notamment dans la politique de gestion des rémunérations.

Il y a d'abord des évolutions automatiques de la masse salariale de l'entreprise, indépendantes de toute décision. Certaines conventions collectives prévoient que le vieillissement du personnel s'accompagne d'une augmentation des niveaux de rémunérations ou de promotions hiérarchiques, elles-mêmes accompagnées de hausses de salaires. Par ailleurs, le départ en retraite constitue un mouvement naturel qui modifie la répartition du personnel par catégorie et l'équilibre des rémunérations. Il va de soi que d'autres évolutions, volontaires celles-là et 
moins directement liées à l'âge, viennent aussi modifier la masse salariale : mouvements d'entrée et de sortie du personnel, changements des formes d'emploi (contrat à durée déterminée, contrat à durée indéterminée, intérim, temps partiel). Tous ces éléments se combinent pour modeler la structure et la dynamique de la masse salariale. Un des résultats immédiats d'une politique de départ des salariés les plus âgés est la réduction de la masse salariale du fait d'un effet de noria positif. Dans ce cas, leur remplacement par un employé plus jeune entraîne immédiatement une baisse de la masse salariale ou de la rémunération moyenne d'une catégorie démographique. L'incidence est encore plus forte en cas d'absence de remplacement; qu'elle touche l'ensemble du personnel ou certaines catégories seulement, les industries où la main-d'œuvre constitue une part importante des coûts fixes (nettoyage, conseil, services, automobile) peuvent y voir un levier d'amélioration rapide de la rentabilité et de la performance financière. Si la logique financière est ici évidente, l'effet de noria est aussi porteur de risques pour l'entreprise car, de façon générale, les salariés les plus âgés sont situés en haut de la pyramide hiérarchique ou très expérimentés. Il est vrai que, de ce point de vue, l'évaluation des retombées négatives sur la performance de l'entreprise est beaucoup plus délicate. La relation entre âge, ancienneté, vieillissement et productivité est, encore une fois, d'une grande complexité.

Elle suppose une évolution du paradigme gestionnaire qui domine de nombreuses politiques de gestion des ressources humaines ${ }^{1}$ : les salariés y sont pensés comme des éléments passifs, le bon fonctionnement de l'entreprise est supposé passer par un contrôle extérieur, le

(I) Daniel Atlan, «1968-2003: les mutations dans la sidérurgie », in Frank Bournois et Pierre Leclair (dir.), Gestion des ressources humaines. Regards croisés en l'bonneur de Bernard Galambaud, Paris, Economica, 2004. rôle de l'encadrement étant de contrôler ce que font les salariés. Une approche modernisée suggère au contraire que les salariés conçoivent et font progresser les processus, et que le rôle de l'encadrement est d'obtenir leur implication. C'est cette mutation « culturelle » qui a été enclenchée chez Usinor dans les années 1980, puis relayée chez Arcelor. Un tel virage suppose une remise en cause des relations déterministes établies par de nombreux gestionnaires entre âge, vieillissement et performances des entreprises, relations susceptibles de produire des solutions contre-productives.

\section{Ambiguités dans l'usage gestionnaire}

La pyramide des âges fournit au décideur une photographie d'un «stock » de population à une date donnée répartie selon une variable, l'âge. Cette représentation fournit-elle pour autant une description pertinente du problème ? La première réaction des dirigeants d'Usinor constitue un exemple du biais que peut engendrer cet outil démographique. En les agglomérant au sein d'une représentation statistique, il compare de façon brute des salariés relevant de différentes générations. Mais qu'y a t-il de commun entre des individus nés dans les années 1950, qui auraient débuté leur carrière en usine durant la croissance économique et sur des outils datant parfois des années 1930 ou 1940, et ceux nés en 1970, embauchés après les grandes restructurations sur des installations souvent modernisées $^{2}$ ?

En fait, le vieillissement d'un groupe démographique résulte de la combinaison de l'augmentation des catégories les plus âgées d'une population et de la diminution du poids des catégories les plus jeunes. Une population vieillit plus vite lorsqu'il y a de moins en moins de jeunes par le simple effet mécanique du

(2) Évelyne Sullerot, L'Âge de travailler, Paris, Fayard, 1986. 
temps qui passe. Dans la plupart des entreprises, c'est donc bien un blocage des embauches de jeunes qui a entraîné l'augmentation progressive de l'âge moyen du personnel. Pour autant, il est impossible de rétablir rapidement la situation par une simple embauche de jeunes : il faut tenir compte des effectifs respectifs de chaque tranche d'âge. Ceci relativise par conséquent les retombées, en matière d'âge, des politiques d'embauches massives.

La prise de conscience du déséquilibre de la pyramide des âges de l'entreprise repose aussi sur une autre forme de raisonnement : la comparaison entre les pyramides des âges de plusieurs entreprises, situées ou non dans la même branche. De la représentation statique qu'est la pyramide des âges, est extrapolée une approche dynamique des évolutions démographiques ${ }^{1}$. Or, par définition, la pyramide des âges est une représentation statique qui évolue, certes, selon une logique quantitative mais aussi qualitative. Peut-on comparer sans précautions les conditions de vieillissement - autrement dit les conditions de travail - des salariés d'une entreprise de haute technologie et celles d'une entreprise du textile? Peut-on comparer sans difficulté les conditions du vieillissement d'un opérateur de ligne dans l'automobile des années 1960 et celle d'un de ses collègues des années 1990 ? Peut-on enfin comparer sans précautions le vieillissement d'une population de cadres administratifs avec celui d'opérateurs de production ? Posée de cette façon, la réponse paraît évidemment négative. La démarche est néanmoins fréquente dans la pratique du benchmarking, qui consiste à étalonner l'entreprise vis-à-vis

(I) Hervé Le Bras, Marianne et les lapins. L'obsession démographique, Paris, Hachette littératures, « Pluriel », 1993. On rencontre souvent ce type de raisonnement en histoire économique ou en politique des retraites. L'observateur déduit alors de la comparaison entre plusieurs pyramides des âges d'un pays des conclusions sur son vieillissement ou sa dénatalité puis les applique à la dynamique économique. Une démarche de même type est menée dans l'entreprise mais à une échelle plus réduite. d'autres sociétés jugées plus performantes dans le même secteur ou dans une économie. Ceci lui permet ensuite de reconstruire ses outils, ses méthodes et ses modèles d'organisation ou de stratégie ${ }^{2}$. Quels sont les risques d'une telle approche ?

En comparant à différentes époques les pyramides d'une même entreprise ou celles de plusieurs entreprises, l'observateur fige l'image des différentes catégories d'âge qui la composent. Du coup, ce sont les conditions économiques, techniques et sociales du vieillissement qui sont homogénéisées de façon artificielle. Une utilisation systématique de ce type de rapprochements aboutit à noyer l'évolution de chaque individu au sein d'une représentation trop globale. En osant un parallèle, comparer les pyramides des âges de différents lieux et de différentes époques revient à aligner un ensemble de photographies instantanées d'une course à pied. On peut à la limite retracer l'histoire de la course mais pas celle de chaque participant. Ce glissement du qualitatif au quantitatif procède d'un oubli. Si la pyramide des âges restitue bien la vision synchronique - c'est-à-dire une représentation des âges et des générations à une date donnée -, il faut souligner que le vieillissement est aussi un phénomène diachronique, qui traduit l'évolution d'un individu ou d'un groupe dans le temps. Par conséquent, deux pyramides calculées à deux dates différentes sur une même population ne sont pas indépendantes mais interdépendantes, ce qui oblige le décideur à prendre en compte la durée. L'outil peut ainsi donner une représentation fausse de la nature des problèmes de l'entreprise. Est-il par ailleurs adapté à une analyse des sources de blocage du changement ou d'inertie de la structure organisationnelle ?

(2) Michael Hammer et James Champy, Le Réengenering, Paris, Dunod, 2003. 


\section{Représentations sur l'âge et le vieillissement}

Comment les représentations les plus fréquentes sur l'âge sont-elles influencées par le recours à la pyramide des âges ? Comment dégager, à partir d'un raisonnement de nature démographique, des conclusions relevant d'un autre champ, pour l'essentiel ici le changement technique ou organisationnel ? Si l'on ne peut nier qu'il existe des relations entre ces domaines, encore convient-il de les préciser. Quelles contraintes pèsent par exemple sur une entreprise confrontée au changement technologique ? Elle doit s'assurer que son savoirfaire et l'expérience accumulés se transmettent des salariés les plus anciens aux salariés les plus jeunes. Par ailleurs, elle doit veiller à ce que ses salariés disposent des compétences nécessaires à la mise en œuvre des nouvelles technologies.

Dans la majorité des cas, les diagnostics et les décisions de gestion sont construits à partir de représentations sociales sur l'âge et le vieillissement largement invalidées par les recherches les plus récentes en sociologie, ergonomie ou gérontologie. Ces travaux prouvent qu'au sein d'un groupe d'individus, le vieillissement est un phénomène de plus en plus hétérogène avec l'avancée de l'âge. Pour de nombreux praticiens d'entreprise, le diagnostic de leur personnel repose sur une représentation très répandue du vieillissement conçu comme un déclin permanent. Peut-on néanmoins réellement établir une relation étroite entre l'âge et les capacités professionnelles d'un salarié ? Sans prétendre résumer ici de très nombreux travaux, deux aspects méritent d'être distingués : les capacités physiques ou physiologiques, et les capacités cognitives ou intellectuelles.

La réduction des capacités physiques avec l'âge apparaît souvent comme la plus évidente aux yeux du grand public. De nombreux ergonomes ont montré les limites de cette repré- sentation ${ }^{1}$. Il n'est pas possible de savoir si des modifications cellulaires et tissulaires ont des conséquences fonctionnelles chez un individu. Il n'y a pas non plus de modèle général d'évolution-détérioration pour l'une ou l'autre des catégories de fonctions (physique, sensorielles, psychomotrice ou mentales); au cours du temps, la forme de l'évolution des capacités fonctionnelles varie, ainsi que l'âge auquel se produit un éventuel déclin pour chaque individu. De surcroît, même lorsque apparaissent des détériorations, les capacités fonctionnelles se transforment et, avec elles, les stratégies d'utilisation des capacités changent. Face à des tâches complexes, les individus les plus âgés utilisent leur expérience pour anticiper les opérations et les changements futurs ce qui, dans certains cas, leur permet de compenser d'éventuelles lenteurs et d'obtenir en définitive des performances proches de celles des plus jeunes ${ }^{2}$. Il est enfin très difficile d'établir une relation linéaire entre l'âge et le vieillissement, parce que les transformations avec l'âge sont différentes d'une personne à l'autre, variant sensiblement selon l'environnement du travail, le milieu social et la formation initiale. Ces éléments permettent de conclure que les salariés n'ont pas l'âge de leurs artères, car les situations individuelles sont de plus en plus diversifiées avec l'avancée de l'âge.

Qu'en est-il des capacités cognitives et intellectuelles ? On utilise souvent l'image d'une trajectoire d'apprentissage passant d'abord par la constitution d'un stock de connaissances durant la formation scolaire initiale, qui s'éroderait au cours de la vie professionnelle. Cette vision, largement répandue parmi les dirigeants d'entreprise, est pourtant démentie par les études

(I) François Desriaux et Catherine Teiger, « L'âge, facteur de sélection au poste de travail », Gérontologie et Société, 45, juillet 1988, p. 33-45.

(2) Jean Poitrenaud, « Structure des aptitudes cognitives et vieillissement », Gérontologie et Société, 2, février 1972, p. 3-85. 
déjà anciennes de Jean Piaget. Ses conclusions soulignent que chaque apprentissage correspond à une restructuration de l'ensemble des connaissances et non à une simple accumulation par strates successives et indépendantes ${ }^{1}$. Les ergonomes ont par la suite affiné ces observations, en démontrant que si les capacités d'apprentissage peuvent s'amoindrir dans le temps (en particulier la mémorisation), les individus les plus âgés réutilisent une intelligence « cristallisée », d'autant plus importante que les connaissances accumulées sont nombreuses et variées. Ils disposent ainsi d'un stock de situations de référence, au sein desquelles ils puisent pour interpréter et réagir à la nouvelle situation. Les récentes recherches sur la construction des compétences professionnelles aboutissent à des conclusions identiques ${ }^{2}$.

Comme ces travaux restent encore peu diffusés dans le monde du management, deux questions sont généralement mises en exergue dans le diagnostic: une population âgée peut-elle s'adapter aux nouvelles technologies? Comment peut-elle transmettre son savoir-faire aux plus jeunes ? Dans certaines entreprises, la réponse se résume au recrutement d'individus jeunes et qualifiés. De fait, il s'agit de réincarner le savoir-faire technologique dans de nouveaux individus. Une telle approche évacue la question fondamentale de la transmission entre les catégories d'âges et plus généralement entre les individus. Gabriel Fragnière précise à propos des modalités de transfert des connaissances entre les générations :

«Il est important de ne pas confondre le problème de l'accumulation des connaissances dans l'histoire qui peut procéder par stockage, oublis, retrouvailles, avec celui du transfert des techniques, qui ne peut procéder que par transmission

(I) Jean Piaget, Le Structuralisme, Paris, PUF, « Que saisje $\gg, 1968$.

(2) Erwan Oiry, De la qualification à la compétence, Paris, L'Harmattan, 2004. directe, sinon c'est le déclin et l'oubli définitif [...]. Cela veut dire que le savoir-faire conditionne l'utilisation des techniques acquises et que le développement de technologies nouvelles disparaît s'il n'est pas "incarné" dans un individu "sachant-faire". Au centre même de la possibilité pour une société ${ }^{3}$ de survivre avec la technologie qui est la sienne, et surtout de la possibilité de se développer, il y a ce que nous appelons aujourd'hui la "formation professionnelle" qui n'est pas autre chose que le processus de réincarnation des savoir-faire dans la génération suivante [...]. Nous dirons qu'une société décline si le système de transmission de ses technologies n'a plus la capacité de les maintenir et de les développer. C'est alors que les outils vieillissent [...]. Avec le développement extrêmement rapide de la technologie, nos systèmes de formation ne sont plus à la page et de ce fait, le transfert de savoir-faire n'est pas encore totalement assuré par les systèmes mis en place pour la société globale ${ }^{4}$. »

La relation entre équilibre des âges et vieillissement s'est ici enrichie de trois éléments : la formation, qui permet la constitution des savoir-faire, le système de transmission entre générations et la rapidité de l'évolution technologique. La structure démographique de la population est loin de constituer la seule explication au blocage des possibilités de changement technologique. Elle pourrait l'être, à la condition que le système de formation de l'entreprise n'assure pas la transmission des compétences entre des salariés âgés marqués par une organisation du travail parcellisée, et des jeunes salariés souvent mieux formés théoriquement mais dépourvus d'expérience de la vie en entreprise.

Une conclusion émerge ici : plus que l'outil « pyramide des âges » lui-même, c'est son usage qui ne permet pas de donner une vision précise des possibilités d'évolution des salariés âgés.

(3) Au sens macrosocial et non de structure juridique d'une entreprise.

(4) Gabriel Fragnière, « Le processus de vieillissement des sociétés », in Évelyne Sullerot (dir.), op. cit. 
Tout au plus peut-elle fournir une image de la répartition des catégories d'âges. Le raisonnement gestionnaire doit donc trouver un outil de description qui autorise une analyse plus pertinente des conditions passées et futures rencontrées par chaque salarié, et qui fournisse des indications sur leurs possibilités d'évolution. $A$ contrario, le concept de cohorte développé par les démographes se révèle ici intéressant pour les praticiens, mais aussi - voire surtout - pour les historiens et les sociologues qui se penchent sur ces questions dans l'entreprise.

\section{Utiliser le concept de cohorte}

La pyramide des âges sous-estime le fait que les différences entre les individus et les générations ne s'expliquent pas seulement par l'âge, mais aussi par l'évolution des niveaux et des structures de formation, des conditions et de l'organisation du travail, de la complexité du système technique de production. La cohorte, en tant que génération «sociologique », semble donc mieux adaptée que la pyramide des âges à l'analyse du changement. Elle permet de souligner le rôle de la durée d'exposition du salarié à certaines conditions de travail et rejoint le concept d'ancienneté. Toutefois, si certaines informations peuvent être fournies par la pyramide des anciennetés, celle-ci s'intéresse plutôt à la durée totale de présence dans l'entreprise, alors que la cohorte permet de se concentrer sur l'ancienneté dans le poste, qui semble beaucoup plus conditionner les possibilités de changement futur.

Dans l'entreprise, la cohorte peut être construite à partir d'un événement fondateur ou explicatif de la vie du salarié : la date d'entrée dans l'entreprise ${ }^{1}$, le lancement d'une nouvelle

(I) Ceci rappelle la pyramide des anciennetés qui fournit une vision plus précise que la pyramide des âges car elle donne une première idée des durées et des trajectoires des salariés. Elle présente néanmoins le risque de voir rejeter les plus anciens en s'appuyant sur l'argument souvent avancé que l'expérience serait un facteur de blocage à la formation et au changement. Ceci reste à démontrer. installation ou d'un atelier, ou la mise en œuvre de certaines technologies ou méthodes d'organisation (informatique, automatismes, démarches de flexibilité ou de qualité, etc.). On pourrait objecter que la cohorte est un outil difficile à construire dans une entreprise, pourtant certaines d'entres elles l'utilisent déjà ${ }^{2}$. Les responsables du personnel peuvent trouver les informations statistiques nécessaires à sa conception au sein des dossiers individuels de leurs salariés.

La cohorte, parce qu'elle autorise une analyse plus précise de l'évolution d'une génération de salariés, permet de comprendre comment le personnel a été modelé par les conditions techniques et sociales du travail, et par là même de bâtir une stratégie de changement en matière de ressources humaines. En distinguant, à l'intérieur des effectifs, des sous-groupes plus homogènes que s'ils étaient classés selon la seule variable de l'âge, on peut circonscrire de façon plus précise les éventuelles sources de blocages du changement. Celles-ci sont déterminées conjointement par l'évolution de l'organisation du travail, qui définit les contraintes, et la trajectoire des salariés, porteurs d'une expérience et d'un ensemble de compétences.

Malgré une évolution progressive, de nombreuses entreprises ne voient pas encore les conséquences du vieillissement sur la gestion du personnel. Lorsqu'il survient, la prise de conscience est souvent brutale chez les responsables sur le terrain et engendre des interrogations chez les dirigeants. Ils cherchent parfois à y remédier de façon rapide, mais les solutions proposées, si elles améliorent la situation financière à court terme de l'entreprise, sont porteuses de difficultés potentielles par la perte de compétences et de motivation. Par ailleurs, elles n'ont qu'un effet limité sur l'âge moyen du personnel.

(2) Jean-Jacques Sylvestre, « Système hiérarchique et analyse sociale », Revue française de gestion, 98, janvier-février 1990, p. $10-17$. 
À la question de savoir jusqu'à quel point les conditions démographiques pèsent sur le changement organisationnel, cet article a répondu par la plus grande prudence. Dans beaucoup d'entreprises, l'exclusion liée à l'âge s'intensifie : on devient donc vieux de plus en plus jeune ! Pourtant, l'âge n'est pas la variable la plus pertinente pour éclairer les décideurs chargés de mettre en œuvre un changement organisationnel et de gérer des sureffectifs. Ses conséquences sont très variables selon les individus. Loin de se réduire à la marque biologique du temps, elles sont largement conditionnées par l'organisation du travail dans l'entreprise. L'idée d'une diminution irrémédiable des capacités d'adaptation due à l'âge est remise en cause, car les individus développent des pratiques de compensation qui en limitent les conséquences les plus néfastes. Les effets dévastateurs de l'âge peuvent être combattus et anticipés par des mesures préventives : à maints égards, l'entreprise a les vieux qu'elle mérite. En n'adaptant pas ses méthodes de gestion des ressources humaines et son organisation du travail, elle risque d'entraîner un « vieillissement » - ou plus exactement une « rigidification »- prématuré de ses salariés, problème qu'il faudra ensuite résoudre dans des conditions souvent plus difficiles socialement et somme toute plus coûteuses.

Un tel constat amène à relativiser l'aide que la pyramide des âges peut apporter à la prise de décision en matière d'analyse et de gestion du changement dans l'entreprise. L'analyse des éventuels facteurs de blocage suppose de remettre dans leur contexte historique et social les conditions de travail rencontrées par les salariés au cours de leur carrière. La prise en compte de la durée milite pour l'utilisation de la pyramide des anciennetés ou, mieux encore, de la cohorte. De plus, la grande variété des modes de vieillissement et de bilan démographique d'une entreprise à l'autre doit inciter à réclamer plus d'autonomie et de patience dans les solutions adoptées. Cela suppose aussi de remettre en cause l'éventuelle existence d'une pyramide des âges idéale : l'équilibre entre les tranches d'âges ne peut être qu'un objectif atteignable dans une perspective dynamique.

En définitive, deux stratégies opposées semblent possibles : un scénario marqué par l'obsolescence accélérée, la concentration de l'activité économique sur les âges les plus jeunes et les plus rentables, une rotation de plus en plus courte du cycle de rentabilisation ; un scénario construit autour d'un recyclage permanent, la possibilité d'exercer plusieurs métiers à la suite, de se reconvertir à tout âge, d'avoir un travail adapté à chaque étape de sa vie même si un petit nombre paraît de fait difficilement récupérable. Dans ce cadre, la gestion prévisionnelle des emplois et des compétences joue un rôle crucial dans la mise en place d'une organisation qui doit permettre une évolution cohérente des qualifications des salariés et de leurs besoins. Les obstacles que rencontrent les jeunes pour accéder à l'emploi semblent prouver que la réalité se situe entre ces deux branches de l'alternative. Le débat se résume bien à une opposition entre deux conceptions du vieillissement : un déclin inexorable ou un processus susceptible de manipulations considérables étalées dans la durée.

Docteur en histoire de l'EHESS et professeur agrégé en sciences de gestion, Éric Godelier est président du département Humanités et Sciences sociales de l'École polytechnique. Il enseigne la stratégie, la sociologie et la théorie des organisations, ainsi que l'histoire des entreprises, et anime une équipe sur l'épistémologie et les méthodologies au sein du Centre de recherche en gestion. Ses travaux portent sur la conception et la diffusion des outils et des modèles de gestion dans une perspective historique et anthropologique. II a récemment publié, avec Gilles Garel, Enseigner le management : méthodes, institutions, mondialisation (Hermes, 2004), ainsi que Usinor-Arcelor: du local au global (Eyrolles, 2006) et La Culture d'entreprise (La Découverte, 2006). (eric.godelier@polytechnique.edu) 